\title{
Rappel de tous les lots non périmés de Solu-Cortef Act-o-Vials avec de l'alcool benzylique 100 et 500 mg
}

\section{Numéro d'autorisation 23533; anciens pharmacodes 1484796, 1484804}

Par la présente, nous vous informons du rappel par Pfizer SA, à titre préventif, des lots mentionnés ci-dessous de Solu-Cortef AoV 100 et 500 mg avec de l'alcool benzylique jusqu'au niveau du commerce de détail. Ce rappel intervient en accord avec Swissmedic, l'Institut suisse des produits thérapeutiques.

\begin{tabular}{ll}
\hline$N^{\circ}$ du lot & Date de péremption \\
\hline CN7189 & $06 / 2022$ \\
\hline AT5357 & $01 / 2022$ \\
\hline AT5438 & $11 / 2022$ \\
\hline X81036 & $01 / 2022$ \\
\hline W49916 & $12 / 2021$ \\
\hline
\end{tabular}

Le rappel est dû à un risque de confusion entre les nouveaux AoV de Solu-Cortef sans alcool benzylique (commercialisés depuis avril 2020) et les anciens AoV de Solu-Cortef avec alcool benzylique, qui présentent des éléments d'embal- lage pratiquement identiques. L'administration intrathécale de Solu-Cortef AoV sans alcool benzylique est autorisée depuis novembre 2020. Solu-Cortef SAB, anciennement utilisé pour l'administration intrathécale, n'est plus disponible depuis février 2021.

Le rappel s'effectue en sens inverse de la voie de livraison habituelle jusqu'au niveau du commerce de détail. Nous vous prions donc de bien vouloir renvoyer la marchandise du commerce de détail d'ici au 30 mai 2021 au plus tard à votre fournisseur.

L'adresse à laquelle les clients directement approvisionnés par Pfizer SA sont priés de renvoyer la marchandise avant le 12 juin 2021 est la suivante: Alloga SA, Service des retours, Buchmattstrasse 10,3400 Berthoud.

Vous recevrez une note de crédit pour la marchandise retournée.

Pour le signalement de tout effet indésirable (EI), Swissmedic encourage les personnes concer- nées à utiliser l'outil de déclaration d'effets indésirables développé à cet effet. L'utilisation de l'Electronic Vigilance System (EIViS) permet de faire la déclaration des El par saisie directe ou par téléchargement de fichier XML. Vous pourrez trouver toutes les informations nécessaires sous www.swissmedic.ch

Nous vous prions de bien vouloir nous excuser pour les désagréments occasionnés par ce rappel et vous remercions de votre soutien.

Pour les demandes médicales, veuillez vous adresser au service d'information médicale de Pfizer (tél. 043495 71 11; e-mail: EUMEDINFO@pfizer.com). Le service clients (tél. 0800562 825/Customer. ch@pfizer.com) se tient à votre disposition pour répondre à toute question complémentaire.

\section{Pfizer SA}

Zurich 